\title{
Candidate Technologies for Ultra-wideband Nonlinear Optical Fibre Transmission System
}

\author{
Lidia Galdino, Daniel Semrau, Polina Bayvel \\ Optical Networks Group, Dept. of Electronic \& Electrical Engineering, University College London, UK \\ l.galdino@ucl.ac.uk
}

\begin{abstract}
This paper discusses the limitations, practicalities and possible technologies for accomplishing high-capacity broadband transmission systems beyond C+L EDFA bandwidth. It also provides a theoretical understanding of the contribution of different noise source limiting the overall system throughput. (c) 2020 The Author(s)
\end{abstract}

OCIS codes: (060.0060) Fiber optics and optical communications; (060.2360) Fiber optics links and subsystems

\section{Introduction}

The challenge of how to maximise the overall capacity of optical communications systems using single mode fibre has been the subject of much investigation over years. The focus has been mainly on maximising the channel spectral efficiency with advanced coding and modulation techniques, optimising the signal-to noise ratio (SNR), as well as expanding the usable optical fibre bandwidth. The paper covers a short description of different noise source limiting the overall transmission system performance and the currently advancements in amplification schemes to maximise data throughput and increase transmission bandwidth beyond $\mathrm{C}+\mathrm{L}$ band EDFA.

\section{Amplification techniques enabling high- capacacity transmission systems}

Several milestones of record data throughput using sin-

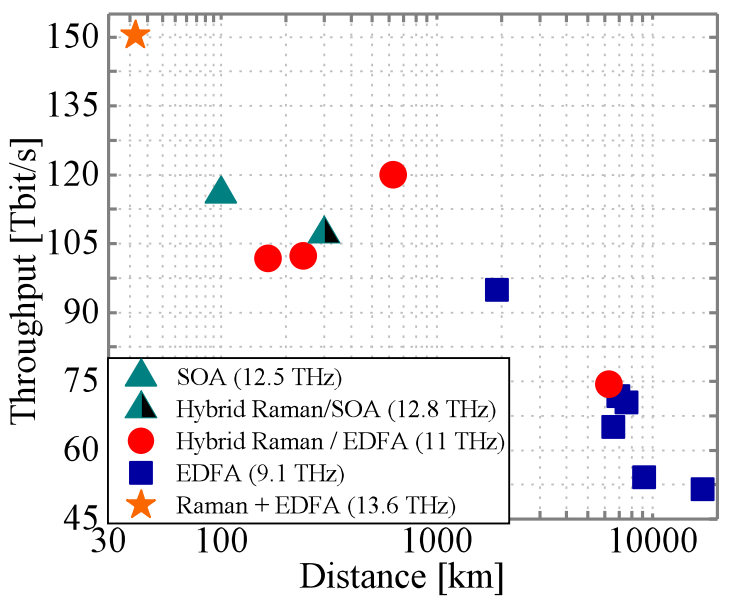

Fig.1: Records data throughput versus distance. gle mode fibre (SMF) [1-12] have been reported over the last few years. Fig. 1, illustrates records data through over distance achieved using different amplification technologies. Aside of [1], where a capacity of $74 \mathrm{Tbit} / \mathrm{s}$ over $6300 \mathrm{~km}$ was achieved using hybrid distributed Raman/EDFA (HRE) amplification scheme, all trans-Atlantic and trans-Pacific record data throughput to date was achieved by using $\mathrm{C}+\mathrm{L}$ band EDFAs. Despite HREs having a lower noise figure compared with EDFAs, this amplification technology is not as power efficient as EDFA systems, which makes it less attractive for long-haul submarine systems that are electrical power feed constrained. C+L band EDFAs systems, empowered by the combination of coded modulation, nonlinearity compensation and per-channel adaptive-rate decoding combined with advanced high speed electronics, large-effective area / low-loss transmission fibres, demonstrated a record capacity of $70.46 \mathrm{Tbit} / \mathrm{s}$ over a trans-Atlantic distance of 7,600 km [2] and record capacity of $51.5 \mathrm{Tbit} / \mathrm{s}$ over a trans-Pacific distance of 17,107 km [4].

On a different approach, the records capacities in short, metropolitan and long-haul transmission distances have been achieved mainly by using amplification technologies that goes beyond $\mathrm{C}+\mathrm{L}$ band EDFAs. In [6], a continuous-band $100 \mathrm{~nm}$ semiconductor optical amplifier (SOA) enabled a potential SMF capacity of $115.9 \mathrm{Tbit} / \mathrm{s}$ over $100 \mathrm{~km}$. Although the bandwidth is notable, SOAs have a relatively high noise figure compared with EDFAs and distributed Raman amplifiers, so the system performance decreases rapidly with distance. Through the combination of SOA and distributed backward Raman amplifier, $107 \mathrm{Tbit} / \mathrm{s}$ transmitted over $300 \mathrm{~km}(3 \times 100 \mathrm{~km})$ was demonstrated in [9]. Higher data throughput of $120 \mathrm{Tbit} / \mathrm{s}$ over $630 \mathrm{~km}(9 \times 70 \mathrm{~km})$ was achieved by using continuous $91 \mathrm{~nm}$ hybrid distributed Raman-EDFA amplifier [10] [11]. Can be noted that by using hybrid Raman/EDFA amplifier, the data throughput and distance are both increased over SOA/Raman amplification scheme, with an extra $13 \mathrm{Tbit} / \mathrm{s}$ capacity over more than 3 times longer transmission distance, whistle using $12 \mathrm{~nm}$ less transmission bandwidth. However, the single model fibre world record capacity of $150.3 \mathrm{Tbit} / \mathrm{s}$ transmitted over $40 \mathrm{~km}$ [12] was 
empowered by extending the transmission bandwidth to S-band wavelengths. It used distributed backward Raman amplification scheme for S-band wavelengths and EDFA for C and L-band wavelengths; with the transmitted signal occupying a total bandwidth of approx. $109 \mathrm{~nm}$.

\section{Transmission system noise source and their relative contributions}

Fundamental limits in signal quality of an optical communication system are imposed by a combination of noise from the transceiver subsystem, optical amplifier and optical fibre nonlinearity. The total signal-to-noise ratio for an optical fibre communication system and a channel of interest $i$ is given by

$$
\mathrm{SNR}_{\mathrm{Total}_{i}}^{-1}=\mathrm{SNR}_{\mathrm{TRX}_{i}}^{-1}+\mathrm{SNR}_{\mathrm{NLI}_{i}}^{-1}+\mathrm{SNR}_{\mathrm{ASE}_{i}}^{-1}
$$

The $\mathrm{SNR}_{\mathrm{ASE}_{i}}$ is a linear noise source generated by optical amplifiers used in the transmission line to compensate for fibre loss. $\mathrm{SNR}_{\mathrm{NLI}_{i}}$ is the nonlinear interference noise generated by fibre nonlinearity and $\mathrm{SNR}_{\mathrm{TRX}_{i}}$ is the transceiver-constrained $\mathrm{SNR}_{\mathrm{TRX}_{i}}$ (i.e. the back-toback SNR). This transceiver noise sets an upper limit on the available SNR, and therefore each channel's greatest achievable information rate. This upper limit on the achievable $\mathrm{SNR}_{\mathrm{TRX}_{i}}$ in a transceiver subsystem is mainly due to the resolution of the digital-to-analog converter (DAC) and analog to digital converter (ADC). The SNR of an ideal DAC / ADC is defined by the effective number of bits (ENOB) which sets the quantisation noise floor [13]. As well as ENOB, other noise sources also constrain the $\mathrm{SNR}_{\mathrm{TRX}_{i}}$. At the transmitter, the linear amplifiers used to drive the IQ-modulator, as well as the TIA amplifiers used to amplify the received signal, both have an associated noise figure which also typically increases with frequency. Furthermore, non-ideal digital signal processing (DSP) at both the transmitter and receiver has an associated penalty that also constrains the transceiver $\operatorname{SNR}_{\operatorname{TRX}_{i}}[14,15]$.

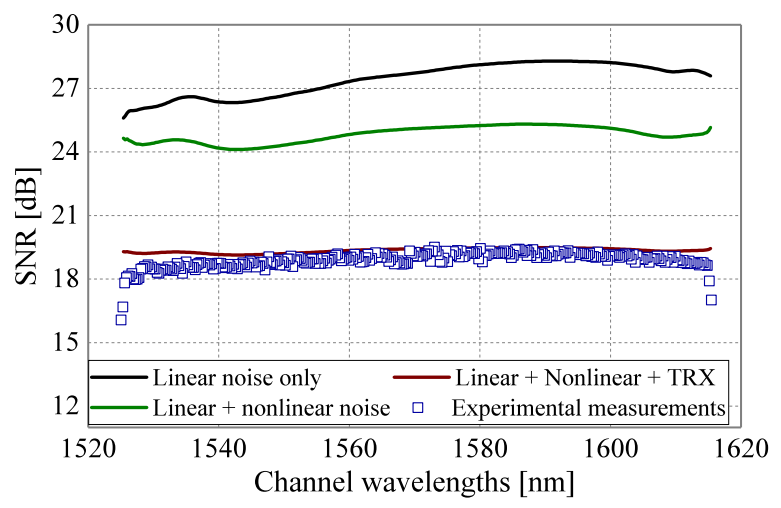

Fig.2: Signal-to-noise ratio including different noise components versus wavelength after $630 \mathrm{~km}$ [11].

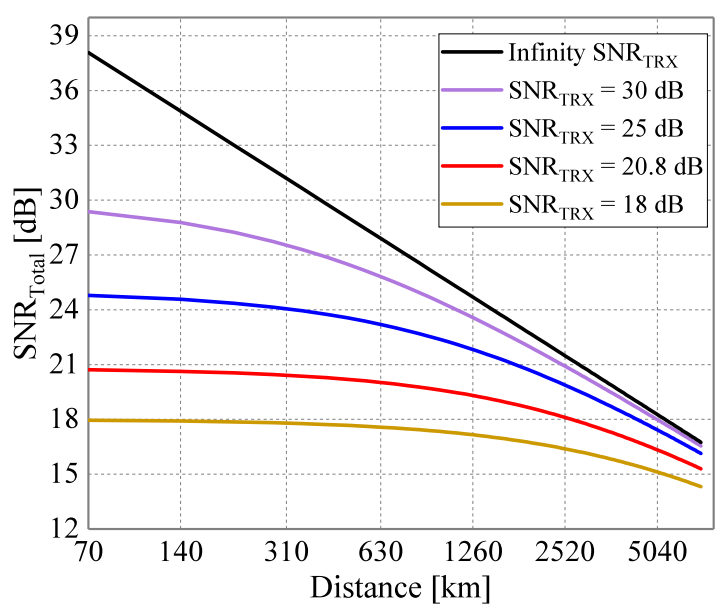

Fig.3: Total signal-to-noise ratio versus distance for different transceiver noise.

To study and quantify the impact of each channel $i$ noise source contribution to the received $\mathrm{SNR}_{\mathrm{Total}_{i}}$, Fig. 2 shows the variation of SNR after $630 \mathrm{~km}$ with channel wavelength. The experimental transmission system under investigation is a $312 \times 35 \mathrm{GBd}$ dual polarization 256-ary quadrature amplitude modulation (DP-256QAM) wavelength division multiplexed (WDM) channels over $9 \times 70 \mathrm{~km}$ single mode fibre spans. The amplification scheme is hybrid distributed Raman-EDFA (HRE) amplifiers with a continuous gain bandwidth of $91 \mathrm{~nm}$. A data throughput of $120 \mathrm{Tbit} / \mathrm{s}$ over a transmission distance of $630 \mathrm{~km}$ is reported in [10,11] for this transmission system under investigation. To estimate the nonlinear noise power per channel $i$, the Gaussian noise model in the presence of inter-channel stimulated Raman scattering [17] was used; details of the system under investigation and modelling can be found in [11]. In Fig. 2 the black line is the SNR calculated assuming the presence of linear noise power only (HRE amplifier noise), the green line shows the $\mathrm{SNR}_{\mathrm{Link}_{i}}$, which takes into account the linear noise power of the in line HRE amplifier and nonlinear noise power from the optical fibre. The brown line shows the received $\mathrm{SNR}_{\mathrm{Total}_{i}}$ taking into account all noise contributions (linear, nonlinear and transceiver). The square blue markers illustrates the experimentally measured $\mathrm{SNR}_{\mathrm{Total}_{i}}$ after $630 \mathrm{~km}$. A penalty in the mean SNR due to nonlinear interference noise was found to be only $2.6 \mathrm{~dB}$, providing a mean link SNR of $24.8 \mathrm{~dB}$. By adding the transceiver noise, the received SNR further decreases to $19.8 \mathrm{~dB}$; a $5 \mathrm{~dB}$ penalty on the $\mathrm{SNR}_{\text {Total }}$ due to the transceiver noise only.

For this specific system and distance, the transceiver constrained-SNR is the major noise source contribution in limiting the signal quality and therefore the data throughput. In order to investigating the implications of transceiver noise on the overall system performance for a range of transmission distances, Fig. 3, shows the ISRS GN-model prediction [17] of the average received $\mathrm{SNR}_{\text {Total }}$ of the 312 channels as a function of distance 
and different transceiver noise contribution. The black line illustrates the model prediction of the mean receiver $\mathrm{SNR}_{\text {Total }}$ when the transceiver subsystem is ideal $\left(\mathrm{SNR}_{\mathrm{TRX}}=\infty\right)$. The other lines illustrate the transmission system performance for a range of transceiver noise. This results indicates that, for short, medium and long-haul distance range (up to $1,000 \mathrm{~km}$ ) the transceiver noise is the predominant noise source on this transmission system under investigation. However for ultra-long haul distances, the system performance becomes dominated by the amplifier and fibre nonlinearity noise.

In order to maximise the transceiver constrained-SNR, it is paramount to understand and quantify it's limitation and noise contributions. Several papers $[14,16]$ have shown that transceiver SNR decreases with the increase of the channel symbol rate. Mainly due to the clock jitter, the ENOB is a function of frequency and is reduced at high frequencies, which consequently diminishes the SNR as the channel symbol rate is increased. Therefore, lower transceiver symbol rates could enable a significant increase in overall data throughput. However, this gain in achievable information rate per wavelength comes with an increase in the number of transceivers required to maximise the use of any given transmission bandwidth, which consequently may impact on the overall cost per bit.

\section{Acknowledgments}

Dr Lidia Galdino is supported by the Royal Academy of Engineering under the Research Fellowships scheme and The Royal Society Research Grant. This work is supported by the EPSRC programme grant EP/R035342/1 "TRANSNET".The authors are grateful to the industrial partner Xtera.

\section{References}

1. M. Ionesco, at. al. "74.38 Tb/s Transmission Over $6300 \mathrm{~km}$ Single Mode Fiber with Hybrid EDFA/Raman Amplifiers”, Proc. OFC, paper Tu3F.3, (2019).

2. T-X. Cai et al., "70.46 Tb/s over 7,600 km in in C+ L Band Using Coded Modulation with Hybrid Constellation Shaping and Nonlinearity Compensation," Proc. OFC, PDP Th5B.2 (2017).

3. A. Ghazisaeidi et al., "Advanced C+L-Band Transoceanic Transmission Systems Based on Probabilistically Shaped PDM-64QAM,” J. Lightw. Technol., 35 (7), (2017).

4. T-X. Cai et al., "51.5 Tb/s Capacity over 17,107 km in C+L Bandwidth Using Single-Mode Fibers and Nonlinearity Compensation," J. Lightw. Technol., 36 (11), (2018).

5. T-X. Cai et al., "49.3 Tb/s Transmission Over $9100 \mathrm{~km}$ Using C+L EDFA and $54 \mathrm{~Tb} / \mathrm{s}$ Transmission Over 9150 km Using Hybrid-Raman EDFA,” J. Lightw. Technol., 33 (13), (2015).

6. J. Renaudier et al., "First 100-nm Continuous-Band WDM Transmission System with 115Tb/s Transport over 100km Using Novel Ultra-Wideband Semiconductor Optical Amplifiers," Proc. ECOC, PDP (2017).

7. A. Sano et al., "102.3-Tb/s C-band extended L-band all Raman transmission over $240 \mathrm{~km}$ using PDM64QAM single carrier FDM with digital pilot tone,” Proc. OFC, PDPSC.3 (2012).

8. D. Qian et al., "101.7-Tb/s (370x294-Gb/s) PDM-128QAM-OFDM Transmission over 3x55-km SSMF using Pilot-based Phase Noise Mitigation,” Proc. OFC, PDPB.5 (2011).

9. J. Renaudier et al., "107 Tb/s Transmission of 103-nm Bandwidth over $3 \times 100 \mathrm{~km}$ SSMF using UltraWideband Hybrid Raman/SOA Repeaters," Proc. OFC, Tu3F.2, (2019).

10. M. Ionescu et al., "90 nm C + L Hybrid Distributed Raman/Erbium-Doped amplifier for High Capacity Subsea Transmission," Proc. ECOC,(2018).

11. L. Galdino, et al., "Study on the Impact of Nonlinearity and Noise on the Performance of High-Capacity Broadband Hybrid Raman-EDFA Amplified System”, J. Lightw. Technol., online version, (2019).

12. F. Hamaoka, et al., "150.3-Tb/s Ultra-Wideband (S, C, and L Bands) Single-Mode Fibre Transmission over 40-km Using ¿519Gb/s/A PDM-128QAM Signals,” Proc. ECOC,(2018). 2018.

13. C. Laperle and M. Sullivan, "Advances in high-speed DACs, ADCs, and DSP for optical coherent transceivers," J. Lightw. Technol., vol. 34, n. 4, 2014.

14. L. Galdino, et al., "The Trade-off Between Transceiver Capacity and Symbol rate," Proc. OFC, p. M2E.6.

15. L. Galdino, et al., "On the limits of digital back-propagation in the presence of transceiver noise", Optics Express, Vol. 25, no. 4, 2017.

16. R. Maher, et al., "Constellation shaping for high symbol rate SNR limited transceivers," Proc. of the SPIE, Vol. 10947, 2019.

17. D. Semrau et al., "The Gaussian Noise Mode in the Presence of Inter-Channel Stimulated Raman Scattering," J. Lightw. Technol., vol. 36, no. 14, 2018. 